Revista Brasileira do Esporte Coletivo - v. 1, n. 2, 2017.

Artigo Original

\title{
NÍVEL DE CONHECIMENTO TÁTICO DECLARATIVO NO FUTEBOL
}

Título resumido:

Conhecimento no futebol

Júlio Cesar Lima'; Nícolas Grigório Miranda².

\section{RESUMO}

Introdução: As modalidades esportivas coletivas apresentam ambientes sempre imprevisíveis, e entender os processos cognitivos é essencial para que as situações problemas sejam solucionadas da melhor forma, permitindo assim um melhor desempenho do atleta no jogo. Objetivo: O objetivo deste estudo foi comparar o nível de conhecimento tático declarativo de jogadores de futebol da categoria infantil masculino, pertencentes a dois clubes de futebol do estado de Pernambuco. Método: O estudo foi do tipo descritivo e transversal com amostras por conveniência. Foram analisados 51 atletas da categoria infantil masculino, pertencentes a dois clubes de futebol da cidade do Recife - PE (Grupo A $n=26$; grupo B $n=25$ indivíduos). Utilizou-se os protocolos de Mangas (1999) com adaptações feitas por Giacomini (2007). Para a análise descritiva dos dados foram utilizadas medidas de tendência central (mediana) e de variabilidade (erro padrão). Para as análises inferenciais, utilizaram-se inicialmente os testes de Bartllet (homogeneidade de variâncias) e de Shapiro Wilk (distribuição normal). Posteriormente, foi empregado o teste de Manny Witney com um nível de significância de $p \leq 0,05$. Os dados foram gerados no pacote estatístico SPSS for Windows 2010, versão 17.0. Resultados: Os resultados obtidos foram: grupo "A" Med = 13,8; $E P=0,48 ; N^{\circ}$ AC Geral= 544. Grupo "B" Med = 13,2; EP=0,54; № AC Geral= 466. Conclusão: Concluímos que o tempo de prática (experiência) influencia nos resultados dos níveis de conhecimento tático declarativo dos atletas e que os protocolos utilizados foram eficientes para o fenômeno verificado.

Palavras-chaves: Esporte, futebol, cognição, conhecimento.

\section{ABSTRACT}

Introdution: The collective sports have always unpredictable environments, and to understand the cognitive processes is essential for problem situations are resolved in the best way, thus allowing a better athlete performance in the game. Objective: The aim of this study was compare the level of declarative tactical knowledge of footballers male child category, belonging to two football clubs in the state of Pernambuco. Method: The study is descriptive and was cross-sectional with samples of convenience. 51 male athletes of the children's category, were analyzed belonging to two football clubs of Recife - PE (Group A $n=26$, group $B n=25$ individuals). We used protocols Mangas (1999) with adaptations made by Giacomini (2007). Measures of central tendency (median) and variability (standard error) were used for descriptive analysis. For inferential analyzes, we used initially tests Bartllet (homogeneity of variances) and Shapiro Wilk (normal distribution). Subsequently, we employed the Manny Witney with a significance level of $p \leq 0.05$. The data were generated in the statistical package SPSS for Windows 2010, version 17.0. Results: The results were: Group "A" Med = 13.8; SE = 0.48; № AC = 544 General Group "B" Med = 13.2; SE $=0.54$; № General AC $=466$. Conclusion: We conclude that the practice time (experience) influences the results of the CTD levels of athletes and the protocols used were efficient for the phenomenon observed.

Keywords: Sport, football, cognition, knowledge.

1 Universidade Federal de Pernambuco, Centro Acadêmico de Vitoria, Núcleo de Educação Física e Ciências do Esporte, Vitoria de Santo Antão, Pernambuco, Brasil.

e-mail: juuliocesarlima@gmail.com

2 Universidade Federal de Pernambuco, Centro Acadêmico de Vitoria, Núcleo de Educação Física e Ciências do Esporte, Vitoria de Santo Antão, Pernambuco, Brasil.

e-mail: niche_1992@hotmail.com

Endereço para correspondência: Universidade Federal de Pernambuco - Centro Acadêmico de Vitoria, Núcleo de Educação Física e Ciências do Esporte. Rua: Alto do Reservatório, S/N, Bela Vista CEP: 55608-680 - Vitoria de Santo Antão, PE - Brasil. Fone: 81-35233351 / 997746783. E-mail: iberecaldas@gmail.com 
NÍVEL DE CONHECIMENTO TÁTICO DECLARATIVO NO FUTEBOL

\section{INTRODUÇÃO}

O esporte é um fenômeno mundial que se caracteriza por atividades físicas ou exercícios físicos que são praticados tendo ou não intuito competitivo e que respeitam regras préestabelecidas (CALDAS, 2014). Esse fenômeno se apresenta na forma individual ou coletiva, onde as modalidades esportivas coletivas (MEC) constituem-se por ações de cooperação, situações de oposição e de invasão do campo adversário, ambientes variáveis e aspectos tático-estratégicos (GARGANTA, 2000).

$\mathrm{Na}$ execução das MEC duas equipes atuam de forma particular, buscando um objetivo (vencer), vivenciando momentos de ataque $\mathrm{e}$ defesa (GARGANTA, 2001). A exemplo de uma modalidade esportiva coletiva, o futebol é um esporte que apresenta essas características, onde em suas estratégias há um objetivo a se obter, a vitória (COSTA et al, 2009). Dessa forma o futebol torna-se um esporte estratégico, imprevisível e aleatório, onde passa exigir de seus praticantes uma elevada capacidade perceptiva durante a prática do mesmo (GARGANTA, 2001).

Durante a prática do futebol, situações problemas são vivenciadas eé requerido do atleta um comportamento adequado que solicitam do mesmo a utilização de processos cognitivos que os levam a tomarem melhores decisões na competição (BANKS; MILLWARD, 2007, MORALES et al., 2012), dando ênfase as estruturas mentais que se relacionam com o conhecimento (GRECO, 2007).

Nas ciências do esporte, o conhecimento aplicado não se apresenta de forma geral e sim específica dentro das MEC, este é definido como conhecimento tático, que segundo BANKS; MILLWARD (2007) esse conhecimento se distingue em dois tipos, o conhecimento tático declarativo (CTD) e o conhecimento tático processual (CTP). No qual o CTD estaria relacionado à capacidade do indivíduo de saber "o que fazer" declarando de forma verbal ou escrita qual decisão a ser tomada e o porquê da mesma, já o CTP consiste no como fazer, ou seja, na operacionalização da ação (MCPHERSON; KERNODLE, 2007).

Esses dois tipos de conhecimentos interagem influenciando como o atleta irá executar ações específicas no jogo, isso, através da compreensão de um cenário específico e mutável constantemente (situação problema) (COSTA et al., 2009; GRECO 2011; MATIAS , GRECO, 2013). Dessa forma como referencia a literatura especializada, o nível do CTD varia a partir da experiência dos atletas e dos anos de prática na modalidade (GIACOMINI, 2011). Levando em consideração a importância do CTD na prática do futebol, o objetivo do nosso estudo foi comparar níveis de CTD de atletas de futebol categoria infantil, do sexo masculino de dois clubes de futebol da Região Metropolitana do Recife - PE.

\section{MATERIAIS E MÉTODOS}

Por meio de um estudo do tipo explicativo e transversal com amostras por conveniência, foram analisadas respostas sobre o nível de conhecimento tático declarativo (CTD) de atletas da categoria infantil masculino, pertencentes a dois clubes de futebol do Estado de Pernambuco, localizados na Região Metropolitana do Recife. A amostra foi composta por 51 indivíduos, sendo estes 26 atletas da equipe A e 25 da equipe B. A coleta dos dados o ocorreu no outubro de 2015. O estudo respeitou as normas estabelecidas pelo Conselho Nacional em Saúde (466/2012) envolvendo pesquisa com seres humanos, sendo aprovado pelo comitê de ética da Universidade Federal de Pernambuco (protocolo $N^{\circ} 94.896$ de 14/08/2012).

Caracterizou-se como critério de inclusão os atletas que estivessem na faixa etária da categoria e atuantes das equipes investigadas. $\mathrm{O}$ objetivo do estudo foi muito bem informado aos profissionais responsáveis pelas equipes, garantindo-lhes total sigilo sobre as informações recolhidas com o teste. Além disso, todos os sujeitos participantes da coleta assinaram um termo de Consentimento Livre e Esclarecido.

Para a coleta dos dados utilizamos os protocolos de Mangas (1999) com adaptações feitas pelo Giacomini (2007). Este protocolo contêm 13 cenas de vídeo de ações ofensivas de jogos de futebol, sendo as duas primeiras cenas de adaptação (treino) para os atletas avaliados. O número de respostas (certas e erradas) permite avaliar o nível de CTD dos participantes.

As imagens do teste do nível de CTD no futebol foram projetadas por um projetor multimídia anexado ao computador, na qual as cenas apresentaram um tempo de duração de aproximadamente 7 à 10 segundos. Ao final de cada cena a mesma era paralisada no momento em que o jogador que estava de posse de bola no ataque onde o indivíduo avaliado decidiria "o que fazer" (decisão). A partir disso surgiram 4 fotos na projeção, numeradas de 1 a 4 , com possíveis soluções para a situação problema da ação tática. Os indivíduos testados tiveram o tempo que julgassem necessário para decidir e anotar suas respostas numa ficha de avaliação (folha resposta). Para a análise descritiva dos dados foi utilizado as medidas de tendência central (mediana) e de variabilidade (erro padrão). Para as análises inferenciais, utilizaram-se inicialmente os testes de Bartllet (homogeneidade de variâncias) e de Shapiro Wilk (distribuição normal). Posteriormente, foi empregado o teste de Manny Witney com um nível de significância de $p \leq 0,05$. Os dados foram gerados no pacote estatístico SPSS for Windows 2007, versão 17.0

\section{RESULTADOS}

Abaixo são descritos os valores obtidos pelos grupos investigados, bem como o grau de significância obtido no teste estatístico. Logo após teremos a discussão desses resultados.

Tabela 1. Comparação do número de acertos de atletas infantis de duas equipes da cidade do Recife submetidos ao teste do nível de CTD no futebol.

\begin{tabular}{lllll}
\hline $\begin{array}{l}\text { GRUPO } \\
\text { (Equipes) }\end{array}$ & Média & EP & $\begin{array}{l}\text { № } \mathrm{AC} \\
\text { geral }\end{array}$ & $\begin{array}{l}{ }^{*} \mathrm{p} \leq 0,05 \\
(1 \times 2)\end{array}$ \\
\hline & & & & \\
1 & 13,8 & 0,48 & 544 & $\mathrm{p} \geq 0,59$ \\
2 & 13,2 & 0,54 & 466 & \\
& & & &
\end{tabular}


Med =Mediana; EP = Erro Padrão; № AC Geral= Número de acerto geral; *Significância estatística $\mathrm{p} \leq 0,05$.

Para a variável que foi proposta, os resultados não apresentaram diferença significativa.

\section{DISCUSSÃO}

Em se tratando do protocolo utilizado para a verificação do fenômeno que foi analisado, o escolhemos devido a sua fidedignidade comprovada pelos resultados já apresentados em outros estudos (IROKAWA, 2011; GIACOMINI, 2007; COSTA, 2002); onde através das adaptações feitas por Giacomini (2007) o mesmo se tornou uma ferramenta importante para a análise proposta.

Em uma primeira análise dos dados e pelo fato dos atletas estarem na mesma faixa etária e pertencerem à mesma categoria, observou-se que os resultados não apresentaram diferenças significativas. Um fator determinante para que os resultados dos níveis de CTD sofram influencia é o tempo de prática (experiência) do indivíduo com a modalidade. Segundo Garganta (2006); Giacomini et al., (2011), o tempo de prática além de ser um fator determinante para obtenção de respostas sobre o nível de CTD dos atletas na modalidade, também é de suma importância para que os mesmos tenham conhecimento sobre seus aspectos cognitivos e aprimorem no treino questões como: percepção, tomada de decisão, antecipação, linguagem, memória e aprendizagem.

Giacomini et al., (2011), propondo suas adaptações na utilização do protocolo de Mangas (1999) aplicou esse teste em atletas das seguintes categorias no futebol; sub $14(X=4,08)$, sub 15 ( $X$ $=5,05)$, sub $17(X=6,34)$ o que corrobora como o nosso estudo no que diz respeito a utilização do mesmo teste, mas os resultados encontrados por esses autores não corroboram com os nossos, onde encontramos resultados medianos superiores para o nível de CTD em atletas da faixa etária sub 15 encontrados por esses autores.

Em outro estudo feito por Giacomini (2007), com uma amostra constituída por atletas da categoria juvenil, esse autor encontrou resultados para os níveis de CTD inferiores $(X=7,28)$ do que encontrados nessa investigação; dessa forma este estudo não corrobora com nosso, pois, embora o tempo de prática (experiência) de atletas juvenis seja maior que o tempo de prática de atletas infantis, quando comparado com essa investigação o nível de CTD dos atletas juvenis foi inferior ao do nosso estudo.

Costa et al., (2002) utilizou o protocolo de Mangas (1999) aplicando esse teste junto a 44 jogadores de futebol categoria juvenil, onde os grupos foram divididos em grupo de nível superior (participavam de campeonato nacional) e o grupo inferior(participam de campeonato distrital). Os resultados mostraram que 0 grupo superior apresentou melhores resultados no que diz respeito ao conhecimento específico do futebol, acerca do número de respostas corretas (Grupo sup. $X=6,59$ $\pm 1,7$; Grupo inf. $X=5,59 \pm 1,73$ ) e número de respostas erradas (Grupo sup. $X=0,50 \pm 0,67$; Grupo inf. $X=0,72 \pm 0,70)$, sendo esse o grupo que possuía maior nível competitivo, corroborando como o nosso estudo no que diz respeito a categoria dos atletas.

Williams et al., (2003), utilizando o TESTFILM (estímulos estruturados e não estruturados em relação ao CTD dos atletas), mostraram que existiu diferença significativa $(p \geq 0,0001)$ entre as respostas de jogadores experientes (idade $X=21,6$ $\pm 1,4$; e_número de jogos $X=670 \pm 156,4)$; para jogadores inexperientes (idade $X=20,8 \pm 2,4$; e número de jogos $X=85 \pm 62,7$ ). Esses resultados demonstram que atletas com idades diferentes mas que atuam na mesma categoria, podem apresentar respostas distintas para testes específicos, permitindo que os mesmos tomem decisões eficientes sobre o CTD no jogo, corroborando novamente com o nosso estudo no que concerne a categoria do atleta e o nível de CTD para o jogo de futebol.

Como a percepção e tomada de decisão das equipes investigadas são aspectos particulares e subjetivos de cada indivíduo, no futuro, teremos que investigar outras variáveis que possam ter associação com o nível de CTD dos atletas, tais como: número de treinos na semana, quantidade de horas treinadas, métodos de ensino aplicados por seus treinadores, entre outros. Diante disso, Mangas (1999), afirma que o número de treinos semanais também é maior quanto mais elevada for à categoria, isto implica em um aumento do contato do atleta com a modalidade, acarretando em um maior desenvolvimento do conhecimento tático do indivíduo.

\section{CONCLUSÃO}

Após a comparação dos níveis de CTD de atletas de futebol em uma mesma categoria, observamos que os grupos não apresentaram diferenças estatísticas significativas. Observou-se também que o tempo de prática e a categoria de jogo, são variáveis determinantes que influenciam diretamente nos resultados dos níveis de CTD dos atletas.

\section{REFERÊNCIAS}

BANKS, A. P. E.; MILLWARD, J. Differentiating Knowledge in Teams: Effect of Shared Declarative and Procedural Knowledge on Team Performance. Group Dynamics, Theory, Research, and Practice, v.11 (2), 95 106, 2007. 
COSTA, I. T.; GARGANTA, J.; FONSECA, A.; BOTELHO, M. Inteligência e conhecimento específico em jovens futebolistas de diferentes níveis competitivos. Revista Portuguesa de Ciências do Desporto, v. 2, n. 4, p. 7-20, 2002.

COSTA, I. T.; GARGANTA, J. M. S.; GRECO, J. P.; MESQUITA, I. Princípios táticos do jogo de futebol: conceitos e aplicação. Revista Motriz, v.15, n.3, p. 657-668, 2009.

GARGANTA, J. O treino da táctica e da estratégia nos jogos desportivos. In: GARGANTA, J. Horizontes e órbitas no treino dos jogos desportivos. Porto: Universidade do Porto - Centro de Estudos dos Jogos Desportivos. 2000. p. 51-61.

GARGANTA, J. A análise da performance nos jogos desportivos: Revisão acerca da análise do jogo. Revista Portuguesa de Ciências do Desporto, v.1, n.1, p. 57-64, 2001.

GARGANTA, J. (Re) Fundar os conceitos de estratégia e táctica nos jogos desportivos colectivos, para promover uma eficácia superior. Revista Brasileira de Educação Física e Esportes, v. 20, p. 201-203. 2006.

GIACOMINI, D. S. Conhecimento tático declarativo e processual no futebol. Estudo comparativo entre jogadores de diferentes categorias e posições. 2007. F. 161. Dissertação (Mestrado em Ciências dos Esportes)-Escola de Educação Física, Fisioterapia e Terapia Ocupacional, Universidade Federal de Minas Gerais. 2007.

GIACOMINNI, D. S.; SOARES, V. O; SANTOS, H. F; MATIAS, C. J; GRECO, P. J. O conhecimento tático declarativo e processual em jogadores de futebol de diferentes escalões. Motricidade, v. 7 (1), p. 43-53.2011.

GRECO, P. J. Tomada de decisão nos jogos esportivos coletivos: o conhecimento tático-técnico como eixo de um modelo pendular. In: Revista Portuguesa de Ciências do Desporto. Porto: Faculdade de Desporto da Universidade do Porto, v. 7, p. 16-16. 2007.

GRECO, P. J. O conhecimento tático declarativo e processual em jogadores de futebol de diferentes escalões. Motricidade, v. 7, n. 1, p. 43-53, 2011.

IROKAWA. A, G. N. Z. Comparação do nível de conhecimento tático declarativo de duas equipes de futebol, relacionado ao tempo de prática do atleta e posição que atua em campo. EFDeportes.com, Revista Digital. Buenos Aires, n. 154. 2011.

MANGAS, CJ. Conhecimento declarativo no futebol: estudo comparativo em praticantes federados e nãofederados, do escalão de sub-14, 117f. 1999. Dissertação (Mestrado em Ciências do Desporto) - Faculdade de Ciências do Desporto e de Educação Física, Universidade do Porto, Porto.

MATIAS, C. J. A. S.; GRECO, P. J. Conhecimento tático declarativo dos levantadores campeões de voleibol. Motriz: Revista de Educação Física, v. 19, n. 1, p. 185-194, 2013.

MCPHERSON, S.; KERNODLE, M. Mapping two new points on the tennis expertise continuum: Tactical skills of adult advanced beginners and entry professionals during competition. Journal Sport Science, v. 25, n. 8, p. 945 959, 2007.

MORALES, J. C. P.; GRECO, P. J.; ANDRADE, R. L. Validade de conteúdo do instrumento para avaliação do conhecimento tático processual no basquetebol. Cuadernos de Psicología del deporte, v. 12, p. 31-36, 2012. 
WILLIAMS, A. M.; WARD, P.; CHAPMAN, C. Training perceptual skill in field hockey: Is there transfer from the laboratory to the field? Research Quarterly for Exercise and Sport, v. 74, n. 1, p. 98-103, 2003. 